\title{
Aspectos genéticos da SAOS
}

\author{
Genetic aspects of obstructive sleep apnea syndrome
}

Adriane C. Mesquita Petruco, Maurício da Cunha Bagnato

\begin{abstract}
Resumo
A fisiopatologia da SAOS é resultante da interação entre fatores genéticos e ambientais. Os mais importantes fatores de risco são obesidade e idade. Outros fatores relevantes são anormalidades craniofaciais, hipotireoidismo, menopausa e uso de álcool e de sedativos. A hereditariedade tem sido relacionada a SAOS pela a associação de SAOS a níveis de HLA, obesidade, síndromes genéticas, etnias, sonolência excessiva, alteração do controle ventilatório, expressão de mediadores inflamatórios, entre outros. Este capítulo aborda a variabilidade genética e fenotípica da doença, demonstrando sua relevância no entendimento da fisiopatologia e na avaliação clínica de SAOS.
\end{abstract}

Descritores: Síndromes da apneia do sono; Apneia do sono tipo obstrutiva/genética; Antígenos HLA.

\begin{abstract}
The physiopathology of obstructive sleep apnea syndrome (OSAS) results from the interaction between genetic and environmental factors. The principal risk factors are obesity and age. Other relevant risk factors are craniofacial abnormalities, hypothyroidism and menopause, as well as the use of alcohol and sedatives. By virtue of its association with factors such as HLA levels, obesity, genetic syndromes, ethnicity, excessive sleepiness, alterations in ventilatory control and expression of inflammatory mediators, OSAS has been related to heritability. This chapter addresses the genetic and phenotypic variability of the disease, showing its relevance in the understanding of the physiopathology and clinical evaluation of OSAS.
\end{abstract}

Keywords: Sleep apnea syndromes; Sleep apnea, obstructive/genetics; HLA antigens.

\section{Introdução}

A fisiopatologia de SAOS não pode ser vista isoladamente como uma disfunção da musculatura de vias aéreas superiores, mas como consequência de um número de patologias inter-relacionadas e fatores de risco, tais como obesidade, idade, menopausa, anormalidades craniofaciais (principalmente envolvendo anormalidades mandibulares), condições congênitas (síndromes de Marfan, de Down e de Pierre Robin), condições adquiridas (hipotireoidismo, acromegalia), ingesta de álcool e sedativos, privação de sono, tabagismo, decúbito dorsal e redução da patência nasal.

Os grandes fatores de risco para SAOS

são a obesidade e a idade. ${ }^{(1)}$

Especula-se que SAOS é dependente, em aproximadamente $40 \%$ dos casos, de uma combinação genética e, em 60\%, de influências ambientais. $^{(2)}$

Há ainda a distinção entre SAOS em adultos e em crianças. A causa mais comum em crianças é a hipertrofia de amígdalas e adenoides que, quando removidas, promove uma melhora de SAOS. Já o papel da obesidade infantil isoladamente é controverso.
0 componente de hereditariedade tem sido reconhecido, mas há dificuldade na elucidação da base genética, devido à heterogeneidade dos fenótipos de SAOS. 0 paradigma atual foi construído como um produto de "fenótipos intermediários" que interagem entre si, a exemplo da morfologia craniofacial, obesidade, suscetibilidade para sonolência diurna, controle ventilatório e controle das vias aéreas superiores. Não se sabe, até o momento, qual componente desse pool tem um papel central, e quais componentes aparecem como epifenômenos. Esses fenótipos intermediários são descritos abaixo. $^{(3)}$

\section{Genes e SAOS}

A SAOS está ligada com vários loci de MHC. Um estudo mostrou um aumento de duas vezes na presença de HLA-A2, havendo correlação desse possivel marcador genético com o grau de obesidade e SAOS (pacientes positivos são mais obesos). A correlação com outros genes também foi demonstrada, tais como HLA-A33, HLA-DRB1*03, DQ-Br*03 e DQ-Br*02 com SAOS, 
assim como HLA-B7, B65, B63, B73 com ronco primário, embora seu significado ainda necessite ser mais bem esclarecido. Da mesma forma, o aumento na frequência do fenótipo Le $(a+b-)$ do grupo sanguíneo de Lewis em roncadores precisa ser mais bem entendido.

Os achados sobre associação entre apoE genótipo E4 e SAOS também são conflitantes. A apoE é uma proteína polimórfica originada de três alelos de um único lócus no cromossomo 19qr3. Embora nenhuma diferença tenha sido encontrada nos níveis de apoE entre pacientes com SAOS e controles, uma proporção maior de homozigotos para o genótipo E4 foi observada no grupo com apneia, mas sem significado estatístico. Em outro estudo, mostrou-se que o risco de $1 \mathrm{AH}>15$ eventos/h de sono era o dobro em pacientes homozigotos para o genótipo E4, independentemente do sexo e IMC.(4)

Também foi relatada a associação entre 0 polimorfismo do gene ligado à enzima conversora de angiotensina (ECA) e a severidade da apneia do sono. Um estudo mostrou que a atividade de ECA está aumentada em pacientes com SAOS, um achado independente da presença de hipertensão arterial, embora a distribuição dos genótipos de ECA e da frequência alélica na apneia obstrutiva do sono não tenha sido diferente daquelas do grupo de pacientes saudáveis. Estudos mais recentes falharam em confirmar a relação entre ECA e IAH ou mesmo entre ECA e SAOS. ${ }^{(5)}$

\section{Obesidade}

A obesidade, sem dúvida, é um dos fatores mais importantes, pois aumenta o risco em 10-14 vezes para SAOS, e a perda de peso reduz o risco para essa condição. ${ }^{(6,7)} A$ deposição de gordura resulta em uma redução do calibre nasofaríngeo que, se significativo, pode levar à hipoventilação devido à reduzida complacência da parede torácica. Além disso, a leptina pode afetar a regulação do centro respiratório. ${ }^{(8)} \mathrm{A}$ hereditariedade para IMC está estimada em 25-40\%, mas uma forte influência ambiental está presente. A suscetibilidade à obesidade é fortemente genética, mas fatores ambientais e hábitos de vida são importantes para a expressão do fenótipo. Existem cerca de 300 marcadores, genes e regiões cromossômicas associados ao fenótipo da obesidade. ${ }^{(9)}$

\section{Sonolência excessiva diurna}

Há diferenças quanto à suscetibilidade à sonolência diurna entre indivíduos com SAOS. Aparentemente, lL-1 e TNF- $\alpha$ têm um papel central nas vias de ativação do sono e de outras citocinas, que incluem $1 \mathrm{~L}-10,1 \mathrm{~L}-6, \mathrm{lL}-2, \mathrm{lL}-4$, IFN, FGF, fator estimulante de colônia de macrófagos, etc. Esses fatores também interagem no desencadeamento do processo de inflamação e da síndrome metabólica. ${ }^{(10,11)}$

\section{Controle de vias aéreas superiores}

0 intervalo para a excitação tônica dos motoneurônios do músculo hipoglosso modula a tendência a colabamento da faringe. 0 receptor para 5HT2A tem se mostrado ser o receptor predominante dos motoneurônios do hipoglosso, porém, com respostas incompletas aos inibidores de recaptação de serotonina. Até o presente, não se conhece o fenótipo resultante da interação genótipo-meio ambiente que possa modular esse tônus muscular. ${ }^{(12)}$

\section{Características familiares e raciais}

A agregação familial é explicada pelo fato de que a maioria dos fatores de risco para o desenvolvimento de SAOS é determinada geneticamente. Estudos com gêmeos monozigóticos mostram maior concordância de ronco em monozigóticos do que em dizigóticos, demonstrando uma herança familiar. ${ }^{(13)}$ Parentes de primeiro grau de pacientes com SAOS relatam mais sintomas de apneia, sonolência excessiva e ronco habitual do que parentes de pacientes controles. $^{(14)}$

0 risco para ronco em parentes de primeiro grau de pacientes com SAOS é três vezes maior, sendo quatro vezes maior se ambos os pais têm história de SAOS. A condição de ser parente de primeiro grau de pacientes com SAOS é considerada um fator de risco para a doença.

Comparando-se a ocorrência de SAOS em famílias de afro-americanos e de europeuamericanos, os afro-descendentes têm maior prevalência, maior gravidade e são acometidos em idade mais jovem. 0 fato pode ser atribuído à anatomia das vias aéreas, que tem maior deposição de tecido mole estreitando a luz da faringe. 0 risco de apneia é duas vezes maior 
para os afro-americanos idosos que para os europeus, além do $1 \mathrm{AH}$ ser maior. ${ }^{(15)}$

Uma forte ligação genética foi demonstrada sobre o IMC em um estudo comparando famílias da Nigéria, Jamaica e África, cuja hereditariedade para o IMC foi de $48-58 \%$. ${ }^{(16)}$

Outro estudo demonstrou que, em famílias afro-americanas, a hereditariedade para $\mathrm{IAH}$ é de $32 \%{ }^{(17)}$

\section{Dismorfismos craniofaciais}

Alterações na morfologia craniofacial interferem na gênese de SAOS de modo determinante. A hipertrofia de amígdalas, palato ogival, posicionamento do osso hioide, alongamento vertical da face, redução das dimensões do diâmetro anteroposterior da base de crânio, macroglossia e micrognatia reduzem a luz das vias aéreas e propiciam o surgimento de SAOS.

Em indivíduos com síndrome de Down, é comum ocorrer SAOS devido a alterações craniofaciais e macroglossia. A síndrome de Marfan, cuja mutação ocorre no gene FBN-1 (responsável pela produção da proteína fibrilina-1), tem alta prevalência de dismorfismo craniofacial, predispondo a SAOS. Um grupo de autores relatou uma prevalência de 64\% de apneia ( $\mathrm{IAH}>5$ eventos/h de sono) nos pacientes com a síndrome. ${ }^{(17)} \mathrm{A}$ síndrome de Pierre Robin cursa com micrognatia, uma situação anatômica que predispõe à apneia obstrutiva do sono. A espondilite anquilosante, doença ligada a HLA-B27, leva a SAOS por vários mecanismos: restrição das vias aéreas por comprometimento da articulação temporomandibular, doença da coluna cervical causando depressão central da respiração e doença pulmonar restritiva. Apresentam SAOS 12\% dos pacientes com espondilite anquilosante, o que pode explicar a sensação de fadiga desses pacientes. ${ }^{(18)}$ A mucopolissacaridose, doença de deposição lisossomal, está associada à significativa morbidade devido às alterações anatômicas e aos distúrbios do sono que ocorrem, principalmente a apneia do sono. Embora não se saiba exatamente a prevalência dessa condição, é necessária a avaliação precoce de crianças com mucopolissacaridose para a redução da morbidade. ${ }^{(19)}$

\section{Alterações no controle ventilatório}

Há uma forte evidência de que fatores genéticos estão associados à magnitude da resposta ventilatória na hipóxia e hipercapnia. Existe um alto grau de hereditariedade quanto à responsividade de quimiorreceptores periféricos à hipóxia e à hipercapnia, mais evidente nos monozigóticos do que nos dizigóticos. ${ }^{(20)}$

Essa anormalidade também foi demonstrada em parentes de primeiro grau de indivíduos com doenças pulmonares. Uma possível sobreposição genética entre SAOS, morte súbita do recémnascido e eventos de aparente risco de vida, combinados com um alto grau de concordância na resposta dos quimiorreceptores observados em gêmeos monozigóticos, enfatizam a importância de fatores genéticos no controle central da ventilação em SAOS.

\section{Mediadores inflamatórios associados a SAOS}

Vários fatores inflamatórios têm sido encontrados em concentração elevada nos indivíduos com SAOS, podendo servir como marcadores biológicos. A endotelina-1 e peptídeos vasoconstritores estão elevados em indivíduos com SAOS e têm seus níveis reduzidos quando esses indivíduos são tratados com CPAP. Citocinas inflamatórias e TNF- $\alpha$ também estão elevados em pacientes com SAOS quando comparados aos controles, assim como os níveis de fibrinogênio e a viscosidade plasmática estão aumentados principalmente pela manhã. ${ }^{(10)}$

\section{Referências}

1. Strohl KP, Redline S. Recognition of obstructive sleep apnea. Am J Respir Crit Care Med. 1996;154(2 Pt 1):279-89.

2. Riha RL, Gislasson T, Diefenbach K. The phenotype and genotype of adult obstructive sleep apnoea/hypopnoea syndrome. Eur Respir J. 2009;33(3):646-55.

3. Riha RL. Genetic aspects of the obstructive sleep apnoea/hypopnoea syndrome--is there a common link with obesity? Respiration. 2009;78(1):5-17.

4. Foley DJ, Masaki K, White L, Redline S. Relationship between apolipoprotein E epsilon4 and sleepdisordered breathing at different ages. JAMA. 2001;286(12):1447-8.

5. Xiao Y, Huang X, Qiu C, Zhu X, Liu Y. Angiotensin 1-converting enzyme gene polymorphism in Chinese patients with obstructive sleep apnea syndrome. Chin Med J (Engl). 1999;112(8):701-4. 
6. Sanders MH, Redline S. Obstructive Sleep Apnea/ Hypopnea Syndrome. Curr Treat Options Neurol. 1999;1(4):279-90.

7. Redline S, Tishler PV, Schluchter M, Aylor J, Clark K, Graham G. Risk factors for sleep-disordered breathing in children. Associations with obesity, race, and respiratory problems. Am J Respir Crit Care Med. 1999;159(5 Pt 1):1527-32.

8. Kapsimalis F, Varouchakis G, Manousaki A, Daskas S, Nikita D, Kryger M, et al. Association of sleep apnea severity and obesity with insulin resistance, C-reactive protein, and leptin levels in male patients with obstructive sleep apnea. Lung. 2008;186(4):209-17.

9. Rankinen T, Zuberi A, Chagnon YC, Weisnagel SJ, Argyropoulos G, Walts B, et al. The human obesity gene map: the 2005 update. Obesity (Silver Spring). 2006;14(4):529-644.

10. Krueger JM, Majde JA. Humoral links between sleep and the immune system: research issues. Ann N Y Acad Sci. 2003;992:9-20.

11. Vgontzas AN, Bixler EO, Chrousos GP. Sleep apnea is a manifestation of the metabolic syndrome. Sleep Med Rev. 2005;9(3):211-24.

12. Fenik P, Veasey SC. Pharmacological characterization of serotonergic receptor activity in the hypoglossal nucleus. Am J Respir Crit Care Med. 2003;167(4):563-9.

13. Ferini-Strambi L, Calori G, Oldani A, Della Marca G, Zucconi M, Castronovo V, et al. Snoring in twins. Respir Med. 1995;89(5):337-40.

14. Redline S, Tosteson T, Tishler PV, Carskadon MA, Millman RP. Studies in the genetics of obstructive sleep apnea. Familial aggregation of symptoms associated with sleep-related breathing disturbances. Am Rev Respir Dis. 1992;145(2 Pt 1):440-4. Erratum in: Am Rev Respir Dis 1992;145(4 Pt 1):979. Milliman RP [corrected to Millman RP].

15. Palmer LJ, Buxbaum SG, Larkin EK, Patel SR, Elston RC, Tishler PV, et al. Whole genome scan for obstructive sleep apnea and obesity in African-American families. Am J Respir Crit Care Med. 2004;169(12):1314-21.

16. Luke A, Guo X, Adeyemo AA, Wilks R, Forrester T, Lowe W Jr, et al. Heritability of obesity-related traits among Nigerians, Jamaicans and US black people. Int J Obes Relat Metab Disord. 2001;25(7):1034-41.

17. Cistulli PA, Sullivan CE. Sleep apnea in Marfan's syndrome. Increased upper airway collapsibility during sleep. Chest. 1995;108(3):631-5.

18. Erb N, Karokis D, Delamere JP, Cushley MJ, Kitas GD. Obstructive sleep apnoea as a cause of fatigue in ankylosing spondylitis. Ann Rheum Dis. 2003;62(2):183-4.

19. Nashed A, Al-Saleh S, Gibbons J, MacLusky l, MacFarlane J, Riekstins A, et al. Sleep-related breathing in children with mucopolysaccharidosis. J Inherit Metab Dis. 2009;32(4):544-50.

20. Thomas DA, Swaminathan S, Beardsmore CS, McArdle EK, MacFadyen UM, Goodenough PC, et al. Comparison of peripheral chemoreceptor responses in monozygotic and dizygotic twin infants. Am Rev Respir Dis. 1993;148(6 Pt 1):1605-9.

\section{Sobre os autores}

\section{Adriane C. Mesquita Petruco}

Médica Responsável pelo Laboratório do Sono de Maringá, Maringá (PR) Brasil.

\section{Maurício da Cunha Bagnato}

Médico Responsável pelo Serviço de Medicina do Sono, Hospital Sírio Libanês, São Paulo (SP) Brasil.

E-mail para contato: clinicabagnato@terra.com.br (M. Bagnato) 